\title{
Pilot study on the effects of the GOLO weight management program and RELEASE supplement on weight and glycemic control in patients with Type 2 Diabetes Mellitus and Obesity
}

\author{
Buynak RJ* \\ Buynak Clinical Research, Valparaiso, IN, USA
}

\begin{abstract}
Objective: This pilot studied assessed the effects of the GOLO Weight Management Program with Release supplement on weight and glycemic control in subjects with type 2 diabetes mellitus and obesity.

Methods: 26 subjects with type 2 diabetes mellitus and obesity were recruited into an open-label study of the GOLO Weight Management System with Release supplement at a single clinical site. 16 subjects completed the study over a 13-week treatment period.

Results: After 13 weeks of open-label treatment, subjects lost an average of $-3.3 \mathrm{~kg}$ (SD 2.47; p <0.001) which represented an average of $-3.53 \%$ (SD 0.02 ) weight loss and was statistically significant. Statistically significant reductions in several body measurement parameters were demonstrated including BMI $-1.17 \mathrm{~kg} / \mathrm{m}^{2}$ $(0.75, \mathrm{p}<0.001)$, waist circumference $-6.41 \mathrm{~cm}(4.22 ; \mathrm{p}<0.001)$, hip circumference $-3.81 \mathrm{~cm}(3.68, \mathrm{p}<0.001)$, and body fat mass $-2.34 \mathrm{~kg}(2.29, \mathrm{p}<0.001)$. Reductions in Hemoglobin A1C averaged $-0.61 \%(0.86 ; p=0.01)$ and reductions in HOMA-IR, a measure of insulin resistance, averaged $-2.25(3.33 ; \mathrm{p}=0.02)$ both statistically significant. Positive trends were observed in reductions of fasting blood glucose [-24.19 mg/dl (52.31), p=0.08] and insulin levels [-2.91 uIU/dl (5.75); p=0.06] that were not statically significant.
\end{abstract}

Conclusions: Treatment with the GOLO Weight Management Program and Release supplement for 13 weeks resulted in weight loss and improvements in glycemic control and insulin resistance in patients with type 2 diabetes mellitus and obesity.

\section{Introduction}

Excess body weight from overeating, poor nutrition and lack of exercise is highly correlated with health conditions [1]. Clinical weight loss in overweight and obese people is associated with improvements in clinical markers of health, including measures of blood sugar metabolism, insulin sensitivity and blood lipids [2].

Populations who are overweight and obese include people who exhibit a heterogeneity in glucose metabolism, ranging from normal to insulin resistant to type 2 diabetes [3]. Elevated body weight and BMI are significant risk factors for the development of insulin resistance and type 2 diabetes mellitus [4]. People who are overweight and obese on this spectrum often have difficulty obtaining meaningful or sustained weight loss. It is well established that people who are insulin resistant or diabetic who lose weight and exercise tend to improve glucose, insulin and lipid metabolism [5].

The Golo Weight Management Program (GWMP) is a commercially available program that includes a diet and exercise plan in addition to a supplement known as Release (www.golo.com). The GWMP is designed to help people who are overweight or obese obtain a balance of nutrients from conventional foods, eat defined portioned meals leading to gradual weight loss and participate in a low-moderate level of daily exercise. The GWMP diet is a point-based system that includes foods from all four of the macronutrient food groups. Daily total caloric intake is based on the individual's sex, age, weight size and activity level. Additionally, the program includes printed instructions, motivation and tips supporting compliance and recommends a minimum of 15 minutes of exercise per day.

The Release supplement contains 7 plant-based ingredients and 3 minerals (Figure 1). Scientific evidence suggests that chromium, zinc and Banaba leaf (Lagerstoemia speciose) extract supplements may have positive effects on glycemic control, lipid metabolism and healthy weight management [6-8].

Banaba leaf is a traditional medicine from southeast Asia which has shown hypoglycemic effects through unknown mechanisms [9]. Zinc is an essential trace mineral that plays an important role in normal islet function and is used in the production of insulin. One mechanism by which zinc may work to control glucose is through supporting insulin signal transduction, which may not function optimally in those with visceral fat [8]. Chromium has been shown to be an important cofactor in the action of insulin [10]. These agents are included in

${ }^{\star}$ Correspondence to: Robert J Buynak, MD, 55 University Suite 106, Valparaiso, IN 46385, USA, Tel: 219-464-8302, E-mail: rbuynak@att.net

Key words: Golo, insulin resistance

Received: March 29, 2019; Accepted: April 11, 2019; Published: April 18, 2019 


\section{Supplement Facts} Serving Size: 1 Capsule Servings Per Container: 90

\begin{tabular}{lcc}
\hline Amount Per Serving & \% Daily Value \\
\hline Proprietary Blend & 297mg \\
\hline $\begin{array}{l}\text { Rhodiola (Rhodiola rosea) root extract (Rhodiolife } \\
\text { [standardized to 3\% rosavins and 1\% salidrosides] }\end{array}$ & $* *$ \\
\hline Inositol & $* *$ \\
\hline $\begin{array}{l}\text { Berberine HCl [from standardized Barberry (Berberis vulgaris) } \\
\text { bark and root extract] }\end{array}$ & $* *$ \\
\hline Gardenia (Gardenia jasminoides) fruit extract 10:1 & $* *$ \\
\hline $\begin{array}{l}\text { Banaba (Lagerstroemia speciosa) leaf extract } \\
\text { [standardized to 18\% corosolic acid] }\end{array}$ & $* *$ \\
Salacia (Salacia reticulata) bark extract 6:1 (Salaretin ${ }^{\circledR}$ ) & $* *$ \\
\hline Apple (Malus domestica) fruit extract [standardized to 75\% polyphenols]** \\
\hline Magnesium (as Albion ${ }^{\circledR}$ dimagnesium malate) & $15 \mathrm{mg}$ & $4 \%$ \\
\hline Zinc (as TRAACS ${ }^{\circledR}$ zinc bisglycinate chelate) & $10 \mathrm{mg}$ & $67 \%$ \\
\hline Chromium (as TRACS ${ }^{\circledR}$ chromium nicotinate glycinate chelate) $70 \mathrm{mcg}$ & $58 \%$ \\
\hline **Daily Value not established
\end{tabular}

Other ingredients: vegetable cellulose (capsule), tapioca dextrin, organic rice concentrate, silica. This product does not contain soy, gluten, dairy, eggs, fish, shellfish, tree nuts, peanuts or wheat.

Figure 1. Package insert release supplement

many supplements sold to people with obesity and diabetes. However, more rigorous studies of the effects of nutritional supplements on these conditions are needed and an impetus for the current study.

In unpublished case studies of clinical and wellness program use in New Zealand, GWMP was shown to reduce body weight in both healthy and diabetic people who were overweight or obese [11]. Secondary endpoints including measures of blood sugar control and lipid metabolism were observed to improve in subjects participating in the GWMP [11].

While the GWMP has been developed and used in both healthy people and people with type 2 diabetes, more research is needed to determine to what extent the program and supplement support healthy weight loss. Given the paucity of research of the validity of most commercial diet programs or over-the-counter supplements, a small pilot study was devised to guide further research into the GWMP and Release supplement. This open-label pilot study was intended to observe the effects of the GWMP and Release supplement in a representative group of subjects with type 2 diabetes mellitus and obesity at one outpatient medical practice.

\section{Methods}

This open-label pilot study evaluating the effect of the GOLO Weight Management Program with Release supplement on weight and indicators of glycemic in subjects with stable type 2 diabetes mellitus and obesity was conducted at one clinical site in the United States from July 2017 to October 2017. The site Buynak Clinical Research in Valparaiso, IN recruited subjects from the practice's patient population or through social media advertisement.

Inclusion criteria were males and females age 18 to 69 with type 2 diabetes mellitus diagnosed for at least 6 months and BMI between 30$45 \mathrm{~kg} / \mathrm{m}^{2}$. Subjects could be on diet therapy or single or combination anti-diabetic medications, although subjects using insulin, or any injectable anti-diabetic medication were excluded. Other exclusion criteria included diagnosis of type 1 diabetes, history of surgical treatments for weight loss or any unstable medical condition. All subjects provided informed consent in the form of a written consent form. The study was approved by Western Institutional Review Board.

The study consisted of 4 visits over approximately 13 weeks. At visit 1 , study eligibility was determined, and subjects were given the commercially available GOLO Weight Management Program and instructed on the program's diet and exercise guidelines (see below). Subjects were given the Release supplement and instructed to take one capsule three times a day with meals. Visit 2 included a telephone call to subjects to assess tolerability of the program. At approximately week 4 , subjects returned for in-office Visit 3 and the final visit 4 occurred at approximately week 13 at the site. At each in-office, visit unused Release supplement tablets were collected, compliance was calculated by pill counting, and open labeled 90-count Release supplement bottles were dispensed as needed.

Body measurements were completed at in-office Visits 1, 3 and 4 and included fasting weight, height, waist and hip circumference, and resting blood pressure and pulse. A Tanita bioimpedance scale (MC$780 \mathrm{U}$ ) was used to measure weight and calculate BMI, body fat and visceral fat. Laboratory evaluations were completed at Visit 1 and Visit 4 which included hemoglobin A1C, fasting insulin, fasting blood glucose, lipid panel (total cholesterol, LDL, HDL, triglycerides). A metabolic panel was also completed at Visit 1 and Visit 4 that included sodium, potassium, chloride, BUN, creatinine, calcium, total protein, albumin, alkaline phosphate, AST, ALT and carbon dioxide. Homeostatic model assessment of insulin resistance HOMA-IR was calculated using the formula [fasting blood glucose $(\mathrm{mg} / \mathrm{dl}) \mathrm{x}$ fasting insulin $(\mathrm{uIU} / \mathrm{ml}) / 405$ [12].

The primary endpoint for the study was change in weight at the end of approximately 13 weeks of treatment with GWMP and openlabel Release supplement. Secondary endpoints included changes in body measurements and body composition analysis, vital signs, A1C, fasting blood glucose, fasting insulin levels and HOMA-IR at the end of 13 weeks of treatment. Additional secondary endpoints included changes in lipid panel and metabolic panel results at the end of the study. Additional safety evaluations included the tracking of adverse events throughout the study period.

Change in the 16 subjects' measurements were analysed over the 13week treatment period. The change between the first and last visit were analysed using repeated measures, two tailed t-tests at a significance level of 0.05 . A total of 26 subjects consented to participate in the study. One subject screen failed for a BMI outside of inclusion criteria.

\section{GOLO weight management program}

At visit 1 subjects were educated on the GWMP diet and exercise plan and were given the same supplemental information given to GOLO commercial customers. Subjects were instructed to select at least one serving from each of 4 food groups (protein, vegetables, carbohydrates and fats) at each of three meals. Protein or protein-carbohydrate snacks were permitted within the subject's daily calorie allotment. Examples of possible serving choices were outlined in the GOLO literature.

The total amount of food that the subject consumed per day was based on the subject's basal metabolic rate and exercise activity. Basal metabolic rates were derived at enrolment through a predetermined conversion based on sex, age, waist size and activity level. Subjects were instructed to add additional calorie allowances based on additional exercise as outlined in the GOLO literature. Daily calorie goals 
Buynak RJ (2019) Pilot study on the effects of the GOLO weight management program and RELEASE supplement on weight and glycemic control in patients with Type 2 Diabetes Mellitus and Obesity

represented an approximately 500 calorie reduction from baseline maintenance levels. Compliance to diet was tracked through subject daily food diaries and subjects' calorie calculations that were later verified by study staff. Exercise was tracked in 15-minute increments in a subject diary.

\section{Results}

The baseline characteristics of the study participates are listed in Table 1. 5 Males and 11 Females completed the study and attended all study visits and were included in the final analysis. 10 subjects withdrew or were removed from the study for the following reasons: 5 lost to follow-up/voluntarily withdrew, 3 to adverse events (2 Loose stool, 1 abdominal pain) and 2 to poor study compliance.

The average age of males participating in the study was 57.8 years and for females 58.5 years. The average starting weight was $93.51 \mathrm{~kg}$ (SD: 13.45 ) and BMI was $34.06 \mathrm{~kg} / \mathrm{m}^{2}$ (3.16) which is considered obese. Average hemoglobin A1c was 7.39 (1.44) and fasting blood glucose was $152 \mathrm{mg} / \mathrm{dl}$ (54.61) indicating poorly controlled type 2 diabetes. Average initial resting systolic blood pressure was $125.38 \mathrm{mmHg}$ (14.78), diastolic blood pressure was $76.38 \mathrm{mmHg}$ (8.55) and LDL cholesterol was $98.19 \mathrm{mg} / \mathrm{dl}$ (36.85). These values were already at or near goal levels in this group of diabetic subjects, primarily due to pre-study treatment with blood pressure and cholesterol medications.

Changes from baseline visit 1 to week 13 in weight, BMI, body analysis and body measurements are listed in Table 2. Overall weight loss averaged $-3.3 \mathrm{~kg}(\mathrm{SD} 2.47 ; \mathrm{p}<0.001)$ which represented an average $-3.53 \%$ (SD 0.02) weight loss and was statistically significant. Reductions in several body measurement parameters were demonstrated including BMI $-1.17 \mathrm{~kg} / \mathrm{m}^{2}(0.75, \mathrm{p}<0.001)$, waist circumference $-6.41 \mathrm{~cm}(4.22$; $\mathrm{p}<0.001)$, hip circumference $-3.81 \mathrm{~cm}(3.68, \mathrm{p}<0.001)$, and body fat mass $-2.34 \mathrm{~kg}(2.29, \mathrm{p}<0.001)$ which were all statistically significant Waist/ Hip Ratio improved by an average of $-0.02(0.04 ; \mathrm{p}=0.03)$. Changes in resting blood pressure and pulse were minimal and not statistically significant as most subjects were pre-treated with anti-hypertensive medication as is standard care in diabetic patients.

Changes from baseline visit 1 to week 13 in markers of glycemic control and insulin sensitivity are listed in Table 3. Reductions in Hemoglobin A1C were statistically significant and averaged $-0.61 \%$ points $(0.86 ; \mathrm{p}=0.01)$ or $8.28 \%$ (0.09) indicating improved glycemic control in the 13-week treatment period. Fasting blood glucose levels changed favorably, $-24.19 \mathrm{mg} / \mathrm{dl}(52.31 ; \mathrm{p}=0.08)$ but were not statistically significant. Insulin levels were reduced by $-2.91 \mathrm{uIU} / \mathrm{L}(5.75 ; \mathrm{p}=0.06)$ indicating a beneficial trend that was not statistically significant. However, HOMA-IR, a standard calculation of insulin resistance, was reduced by an average of $-2.25(3.33 ; \mathrm{p}=02)$ which represented an improvement of $-35.64 \%$ (0) that was statically significant.

Changes from baseline visit 1 to week 13 in lipids and hsCRP are shown in Table 4 . While no changes in lipids were statistically significant, treated subjects demonstrated favorable trends in all facets: total cholesterol reduction, LDL reduction and HDL addition. Triglycerides exhibited the largest change during the study with an average reduction of $-21.81 \mathrm{mg} / \mathrm{dl}(59.66 ; \mathrm{p}=0.16)$ showing a positive trend. Levels of hsCRP, a marker of general inflammation associated with cardiovascular risk, also demonstrated modest average reductions of $-0.15 \mathrm{mg} / \mathrm{l}(1.58 ; \mathrm{p}=0,71)$ that were not statistically significant.

In terms of safety, no significant change in other laboratory findings were observed (Table 5). Three subjects terminated from the study due
Table 1. Baseline characteristics

\begin{tabular}{|c|c|}
\hline Parameter & Value \\
\hline Weight (kg.) & $93.51(13.45)$ \\
\hline BMI (kg/m2) & $34.06(3.16)$ \\
\hline Body Fat (\%) & $38.0(6)$ \\
\hline Body Fat (kg) & $35.16(5.04)$ \\
\hline Muscle (kg) & $55.41(11.94)$ \\
\hline Muscle (\%) & $59.0(5)$ \\
\hline Visceral Fat (kg) & $13.5(4.44)$ \\
\hline Waist $(\mathrm{cm})$ & $113.22(8.84)$ \\
\hline Hips $(\mathrm{cm})$ & $114.97(7.11)$ \\
\hline Waist/Hip & $0.99(0.04)$ \\
\hline Waist/Height & $1.76(0.13)$ \\
\hline BP (systolic) (mmHg) & $125.38(14.78)$ \\
\hline BP (diastolic) (mmHg) & $76.38(8.55)$ \\
\hline Pulse (bpm) & $71.94(10.7)$ \\
\hline Total Cholesterol (mg/dl) & $176.25(45.35)$ \\
\hline $\operatorname{LDL}(\mathrm{mg} / \mathrm{dl})$ & $98.19(36.85)$ \\
\hline HDL (mg/dl) & $44.56(8.8)$ \\
\hline Triglycerides (mg/dl) & $176.12(94.25)$ \\
\hline FBG (mg/dl) & $152.0(54.61)$ \\
\hline $\mathrm{A} 1 \mathrm{C}(\%)$ & $7.39(1.44)$ \\
\hline Insulin (uIU/l) & $15.47(7.66)$ \\
\hline hsCRP (mg/l) & $5.18(3.63)$ \\
\hline HOMA-IR & $6.31(4.59)$ \\
\hline
\end{tabular}

to gastrointestinal adverse events including loose bowel movements or abdominal cramps. No serious adverse events were identified.

\section{Discussion}

In this study, weight loss and improvements in glycemic control and insulin resistance were demonstrated in a group of subjects with type 2 diabetes mellitus and obesity treated with the GWMP and Release supplement for 13 weeks. Among the 16 subjects that completed the study, weight loss averaged $-3.3 \mathrm{~kg}$ (SD 2.47; $\mathrm{p}<0.001$ ) which represented an average of $-3.53 \%$ (SD 0.02) weight loss. Of note, subjects demonstrated reduced fat mass of $-2.34 \mathrm{~kg}(2.29 ; \mathrm{p}<0.001)$ or an average $-6.66 \%(0.07)$ preferentially over muscle mass of $-0.91 \mathrm{~kg}(1.78$; $\mathrm{p}=0.06)$ or $-1.65 \%(0.04)$. In addition, circumference measurements improved preferentially at the waist over the hips with a reduction in waist-hip ratio of $-0.02(0.04 ; p=0.03)$ that was statistically significant. Elevated waist-hip ratio has been noted as an independent risk factor of cardiovascular disease [13].

Weight loss in subjects being treated for type 2 is often difficult to achieve, and treatments for type $2 \mathrm{DM}$ often are often associated with weight gain [14]. One explanation for the weight gain usually seen in diabetic patients is that as blood sugar control is improved with intervention, less glucose is generally lost through renal oversaturation (glycosuria), retaining these calories, and causing the weight gain [14]. The finding of weight loss in this small group of subjects when the GWMP with Release supplement was added to their existing antidiabetic regimen provides evidence in support of the product's use in this population.

Treatment with the GWMP with Release supplement for 13-weeks also resulted in improvements in glycemic control as measured by Hemoglobin A1C. While the initial average A1C of study subjects at baseline (7.5) indicated poor diabetic control, the average A1C level at week 13 (6.8) met the goal A1C level recommended for diabetic patients (less than 7.0) [5]. The exact contribution to glycemic improvements 
Buynak RJ (2019) Pilot study on the effects of the GOLO weight management program and RELEASE supplement on weight and glycemic control in patients with Type 2 Diabetes Mellitus and Obesity

Table 2. Changes from Baseline in Weight and Body Measurements

\begin{tabular}{|c|c|c|c|c|c|}
\hline Parameter & Week One Mean (SD) & Week Thirteen Mean (SD) & Mean Difference (SD) & Mean Difference \% (SD) & P value \\
\hline Weight (kg.) & $93.51(13.45)$ & $90.21(12.66)$ & $-3.31(2.47)$ & $-3.53(0.02)$ & $<0.001$ \\
\hline BMI (kg/m2) & $34.06(3.16)$ & $32.88(3.15)$ & $-1.17(0.75)$ & $-3.45(0.02)$ & $<0.001$ \\
\hline Body Fat (\%) & $38.04(5.73)$ & $36.78(6.33)$ & $-1.26(0.02)$ & $-3.32(0.06)$ & 0.03 \\
\hline Body Fat (kg) & $35.16(5.04)$ & $32.82(5.53)$ & $-2.34(2.29)$ & $-6.66(0.07)$ & $<0.001$ \\
\hline Muscle (kg) & $55.41(11.94)$ & $54.49(11.53)$ & $-0.91(1.78)$ & $-1.65(0.04)$ & 0.06 \\
\hline Muscle (\%) & $58.83(5.49)$ & $60.04(6.08)$ & $1.21(0.02)$ & $2.05(0.04)$ & 0.03 \\
\hline Visceral Fat (kg) & $13.5(4.44)$ & $12.56(4.32)$ & $-0.94(0.93)$ & $-6.94(0.07)$ & $<0.001$ \\
\hline Waist (cm) & $113.22(8.84)$ & $106.81(8.15)$ & $-6.41(4.22)$ & $-5.66(0.04)$ & $<0.001$ \\
\hline Hips (cm) & $114.97(7.11)$ & $111.16(6.25)$ & $-3.81(3.68)$ & $-3.32(0.03)$ & $<0.001$ \\
\hline Waist/Hip & $0.99(0.04)$ & $0.96(0.06)$ & $-0.02(0.04)$ & $-2.23(0.04)$ & 0.03 \\
\hline BP (systolic) (mmHg) & $125.38(14.78)$ & $126.75(10.27)$ & $1.38(12.05)$ & $1.1(0.1)$ & 0.65 \\
\hline BP (diastolic) (mmHg) & $76.38(8.55)$ & $75.56(7.53)$ & $-0.81(6.45)$ & $-1.06(0.09)$ & 0.62 \\
\hline Pulse (bpm) & $71.94(10.7)$ & $70.81(8.63)$ & $-1.12(6.71)$ & $-1.56(0.1)$ & 0.51 \\
\hline
\end{tabular}

Table 3. Changes from Baseline in Markers of Glycemic Control and Insulin Resistance

\begin{tabular}{|c|c|c|c|c|}
\hline Parameter & Week One Mean (SD) & Week Thirteen Mean (SD) & Mean Difference (SD) & Mean Difference \% (SD) \\
\hline FBG (mg/dl) & $152(54.61)$ & $127.81(39.39)$ & $-24.19(52.31)$ & $-15.91(0.31)$ \\
\hline A1C (\%) & $7.39(1.44)$ & $6.78(1.01)$ & $-0.61(0.86)$ & $-8.28(0.09)$ \\
\hline Insulin (uIU/l) & $15.47(7.66)$ & $12.56(4.11)$ & $-2.91(5.75)$ & $-18.83(0.44)$ \\
\hline HOMA-IR & $6.31(4.59)$ & $4.06(2.11)$ & $-2.25(3.33)$ & 0.08 \\
\hline
\end{tabular}

Table 4. Changes from Baseline in Lipid Panel and hsCRP

\begin{tabular}{|c|c|c|c|c|c|}
\hline Parameter & Week One Mean (SD) & Week Thirteen Mean (SD) & Mean Difference (SD) & Mean Difference \% (SD) & P value \\
\hline $\begin{array}{c}\text { Total Cholesterol } \\
\text { (mg/dl) }\end{array}$ & $176.25(45.35)$ & $173.5(47.22)$ & $-2.75(32.94)$ & $-1.56(0.18)$ & 0.74 \\
\hline $\begin{array}{c}\text { LDL } \\
(\mathrm{mg} / \mathrm{dl})\end{array}$ & $98.19(36.85)$ & $97.31(36.87)$ & $-0.88(6.45)$ & $-0.89(0.25)$ & 0.90 \\
\hline $\begin{array}{c}\text { HDL } \\
\text { (mg/dl) }\end{array}$ & $44.56(8.8)$ & $45.31(6.23)$ & $0.75(4.85)$ & $1.68(0.11)$ & 0.55 \\
\hline $\begin{array}{l}\text { Triglycerides } \\
\text { (mg/dl) }\end{array}$ & $176.12(94.25)$ & $154.31(65.24)$ & $-21.81(59.66)$ & $-12.38(0.24)$ & 0.16 \\
\hline $\begin{array}{c}\text { hsCRP } \\
\text { (mg/l) }\end{array}$ & $5.18(3.63)$ & $5.03(4.04)$ & $-0.15(1.58)$ & $-2.85(0.25)$ & 0.71 \\
\hline
\end{tabular}

Table 5. Changes from Baseline in Metabolic Panel and Sex Hormones

\begin{tabular}{|c|c|c|c|c|c|}
\hline Parameter & Week One Mean (SD) & Week Thirteen Mean (SD) & Mean Difference (SD) & 95\% Confidence Interval & P value \\
\hline Sodium $(\mathbf{m E q} / \mathbf{L})$ & $140.44(1.9)$ & $140.56(2)$ & $0.12(2.75)$ & $0.09(0.02)$ & 0.86 \\
\hline Potassium (mEq/L) & $4.46(0.47)$ & $4.38(0.45)$ & $-0.08(0.25)$ & $-1.68(0.06)$ & 0.26 \\
\hline Chloride (mEq/L) & $102.88(2.09)$ & $104(2.07)$ & $1.12(2.83)$ & $1.09(0.03)$ & 0.13 \\
\hline BUN (mg/dl) & $17.19(5.14)$ & $16.94(6.27)$ & $-0.25(3.68)$ & $-1.45(0.22)$ & 0.79 \\
\hline Creatinine (mg/dl) & $0.85(0.18)$ & $0.84(0.19)$ & $-0.01(0.11)$ & $-0.74(0.14)$ & 0.82 \\
\hline Calcium (mg/dl) & $9.84(0.46)$ & $9.92(0.39)$ & $0.08(0.34)$ & $0.8(0.03)$ & 0.36 \\
\hline Total Protein (g/dl) & $7.05(0.45)$ & $7.03(0.33)$ & $-0.02(0.29)$ & $-0.27(0.04)$ & 0.80 \\
\hline Albumin (g/dl) & $4.39(0.29)$ & $4.41(0.26)$ & $0.03(0.16)$ & $0.57(0.04)$ & 0.53 \\
\hline Tot Billi (mg/dl) & $0.56(0.21)$ & $0.59(0.19)$ & $0.03(0.12)$ & $4.44(0.24)$ & 0.43 \\
\hline Alk Phosphate (U/L) & $82.62(17.33)$ & $85.12(23.37)$ & $2.5(13.8)$ & $3.03(0.16)$ & 0.48 \\
\hline $\operatorname{AST}(\mathbf{U} / \mathbf{L})$ & $28.12(21.29)$ & $24.69(20.29)$ & $-3.44(26.72)$ & $-12.22(0.49)$ & 0.61 \\
\hline ALT (U/L) & $34.44(23.2)$ & $29.93(19.97)$ & $-4.51(26.74)$ & $-13.09(0.39)$ & 0.51 \\
\hline Carbon Dioxide (mEq/L) & $26.94(1.72)$ & $26.62(2.15)$ & $-0.32(1.81)$ & $-1.21(0.07)$ & 0.48 \\
\hline WBC $\left(10^{\wedge} 3 /\right.$ ul $)$ & $7.14(2.11)$ & $6.88(1.93)$ & $-0.26(1.28)$ & $-3.67(0.17)$ & 0.43 \\
\hline HGB (g/dl) & $13.76(2.73)$ & $11.96(4.41)$ & $-1.8(4.86)$ & $-13.06(0.63)$ & 0.16 \\
\hline Testosterone (ng/dl) & $313.86(108.61)$ & $319.65(71.51)$ & $5.79(99.65)$ & $1.84(0.44)$ & 0.49 \\
\hline Estradiol MALE (pg/ml) & $32.99(20.65)$ & $33.02(6.26)$ & $0.03(24.56)$ & \#VALUE! & 0.81 \\
\hline PSA $(\mathrm{ng} / \mathrm{ml})$ & $0.55(0.17)$ & $0.58(0.32)$ & $0.03(0.19)$ & $5.23(0.31)$ & 0.75 \\
\hline Progesterone(ng/ml) & $0.99(2.79)$ & $0.21(0.25)$ & $-0.78(2.8)$ & \#VALUE! & 0.38 \\
\hline Estradiol FEMALE (pg/ml) & $30.52(40.85)$ & $13.43(17.68)$ & $-17.09(34.39)$ & \#VALUE! & 0.13 \\
\hline
\end{tabular}


Buynak RJ (2019) Pilot study on the effects of the GOLO weight management program and RELEASE supplement on weight and glycemic control in patients with Type 2 Diabetes Mellitus and Obesity

from changes in diet, weight loss or components of Release supplement was not determined in this study and may be multifactorial.

Glycated hemoglobin A1c (HbA1c) is a marker long used by physicians to measure patients' glucose levels over the previous 8-12 weeks. For this reason, $\mathrm{HbAlc}$ is generally considered a more reliable marker than fasting blood glucose to reflect average levels of blood glucose [5]. Thus, a reduction in $\mathrm{HbAlc}$, along with improvements in body weight, may indicate an improvement in metabolic function.

This study also demonstrated a significant improvement in insulin resistance as measured by HOMA-IR in these subjects treated with the GWMP with Release supplement for 13-weeks. Elevated levels of fasting insulin and HOMA-IR are hallmarks of type 2 diabetes, and elevated HOMA-IR serves as an independent risk factor for cardiovascular disease. Although reductions in fasting insulin levels in this study were not statistically significant, the trend was favorable. However, reductions in HOMA-IR averaged $-35.64 \%$ (0), a clear demonstration of improvement in insulin resistance.

Commonly used anti-diabetic medications that effect insulin sensitivity are pioglitazone and metformin [15]. The reductions in HOMA-IR demonstrated in this study approximated that seen with these prescribed medications. In this comparable study, pioglitazone reduced HOMA-IR by $39 \%$ which was statically significant, while metformin reduced HOMA-IR by $25 \%$ and was not statistically significant [15]. The components of the GWMP that are responsible for the improvement in HOMA-IR have not been clearly identified. Weight loss itself can lower HOMA-IR levels, although not to the extent seen in this study [16]. This study indicates the components of the Release supplement may play a role in reducing insulin resistance and are possibly additive to that achieved by diet alone.

The significant improvements in HOMA-IR demonstrated by the GWMP system with the Release supplement suggest a beneficial role in other disease states including the Metabolic Syndrome or PCOS. In these disorders, insulin resistance is generally a major component and treatment of the insulin resistance improves clinical outcomes $[17,18]$. More studies would be needed of the GWMP with Release in these individual areas of interest.

Favourable changes to other laboratory tests were observed from baseline visit 1 to week 13 which were not statistically significant. Improvement in liver transaminase enzymes [AST decreased by $-12.22 \%$ (SD $0.49 ; \mathrm{p}=.61$ ) and ALT decreased by $-13.09 \%(0.39$; $\mathrm{p}=0.51)$ ] are often seen with weight loss and most likely reflect decreased inflammation from fatty liver [19]. Changes in female sex hormones are also seen following weight loss [estradiol decreased by $-127.21 \%(-56.72) \mathrm{p}=0.13$ in women] and may represent decrease peripheral fat conversion of hormone pre-cursors [20-21]. No other significant changes in metabolic panel values or other safety variables were observed.

The study was limited by selection of subjects at only one clinical site. In addition, the study was open-label and lacks the rigor of a double-blinded placebo-controlled study. Poor subject compliance with the GWMP diet and exercise component may also have influenced the results of the study. Although compliance with the Release supplement as obtained by pill counts ( 95 percent overall compliance by pill count) was excellent, compliance with diet and exercise recommendations was variable and more difficult to quantify. The relatively high drop-out rate $(10 / 25-40 \%)$ of the subjects consented for the study reflect this difficulty in obtaining compliance with the program. Frustration with previous diet plans and unrealistic expectations about lifestyle change likely contributed to drop outs. In addition, enrolling a population with type 2 diabetes that likely has failed other attempts at diet, exercise and medical therapy presented challenges to compliance.

This study was not designed to elucidate which components of the GWMP and Release supplement were responsible for clinical improvements and may be multifactorial. This study serves a basis for the completion of larger studies in these areas.

\section{Conclusion}

In summary, the GOLO Weight Management Program with Release supplement demonstrated weight loss and improvement in glycemic control when added to standard anti-diabetic medications in this small, single center pilot study. Improvements in insulin resistance as measured by HOMA-IR approximated those seen by existing antidiabetic medications. Further studies will be needed to evaluate the role of the GWMP with Release supplement in diabetic and non-diabetic populations in these and other clinical measures.

\section{Acknowledgement}

The author thanks Jacob Hoffman, BS/BA for statistical analysis.

\section{Conflict of interest}

The author receives funding from GOLO, LLC per a research and consulting agreement, ClinicalTrials.gov Identifier NCT03478202

\section{Data sharing plan}

All the individual data collected during the trial will be available, after declassification, in addition to the study protocol, informed consent form and clinical study report immediately following the publication, ending in 36 months, to researchers who provide a methodologically sounds proposal to achieve aims in the approved proposal. Proposals should be directed to rbuynak@att.net. Data are available only in hard copy format.

\section{References}

1. Gavin JR, Rodbard HW, Fox KM, Grandy S (2009) Association of overweight and obesity with health status, weight management, and exercise behaviors among individuals with type 2 diabetes mellitus or with cardiometabolic risk factors. Risk Manag Healthc Policy 2: 1-7. [Crossref]

2. Hainer V, Toplak H, Mitrakou A (2008) Treatment modalities of obesity: what fits whom? Diabetes Care 31: 269-277. [Crossref]

3. JP Després, I Lemieux (2006) Abdominal obesity and metabolic syndrome. Nature 200 881-887. [Crossref]

4. Narayan KM, Boyle JP, Thompson TJ, Gregg EW, Williamson DF (2007) Effect of BMI on lifetime risk for diabetes in the U.S. Diabetes Care 30: 1562-1566. [Crossref]

5. [No authors listed] (2017) Standards of medical care in diabetes-2017: Diabetes Care 40: S4-S5. [Crossref]

6. Althuis MD, Jordan NE, Ludington EA, Wittes JT (2002) Glucose and insulin response to dietary chromium: a meta-analysis. Am J Clin Nutr 76: 148-155. [Crossref]

7. Judy WV (2003) Antidiabetic activity of a standardized extract (Glucosol) from Lagerstroemia speciose leaves in type 2 diabetics. A dose-dependence study. $J$ Ethopharmacol 87:115-117. [Crossref]

8. Jayawardena R, Ranasinghe P, Galappatthy P, Malkanthi R, Constantine G, et al. (2012) Effects of zinc supplementation on diabetes mellitus: a systematic review and metaanalysis. Diabetol Metab Syndr. 4: 13. [Crossref]

9. Miura T (2012) Management of diabetes and its complications with banaba (Lagerstroemia speciosa L.) and Corosolic Acid. Evid Based Complement Alternat Med 2012: 871495. [Crossref]

10. Cefalu WT, Hu FB (2004) Role of chromium in human health and in diabetes. Diabetes Care 27: 2741-2751. [Crossref] 
Buynak RJ (2019) Pilot study on the effects of the GOLO weight management program and RELEASE supplement on weight and glycemic control in patients with Type 2 Diabetes Mellitus and Obesity

11. Pilot studies on the efficacy of a diet program on body weight in overweight and obese South Africans.

12. Matthews DR, Hosker JP, Rudenski AS, Naylor BA, Treacher DF, et al. (1985) Homeostasis model assessment: insulin resistance and beta-cell function from fasting plasma glucose and insulin concentrations in man. Diabetologia 28:412-419. [Crossref]

13. Czernichow S, Kengne AP, Stamatakis E, Hamer M, Batty GD (2011) Body mass index, waist circumference and waist-hip ratio: which is the better discriminator of cardiovascular disease mortality risk? evidence from an individual-participant meta-analysis of 82864 participants from nine cohort studies. Obes Rev 12: 680-687. [Crossref]

14. Provilus A, Abdallah M, McFarlane S (2011) Weight gain associated with antidiabetic medications. Therapy 8: 113-120.

15. Eguchi K (2007) Comparison of the effects of pioglitazone and metformin on insulin resistance and hormonal markers in patients with impaired glucose tolerance and early diabetes. Hypertens Res 30: 23-30. [Crossref]

16. Clamp LD, Hume DJ, Lambert EV, Kroff J (2017) Enhanced insulin sensitivity in successful, long-term weight loss maintainers compared with matched controls with no weight loss history. Nutr Diabetes 7: e282. [Crossref]
17. Lankarani M, Valizadeh N, Heshmat R, Peimani M, Sohrabvand F (2009) Evaluation of insulin resistance and metabolic syndrome in patients with polycystic ovary syndrome. Gynecological Endocrinology 25: 504-507. [Crossref]

18. Caserta D, Adducchio G, Picchia S, Ralli E, Matteucci E, et al. (2014) Metabolic syndrome and polycystic ovary syndrome: an intriguing overlapping. Gynecol Endocrinol 30: 397-402. [Crossref]

19. Gasteyger C, Larsen TM, Vercruysse F, Astrup A (2008) Effect of a dietary-induced weight loss on liver enzymes in obese subjects. Am J Clin Nutr 87: 1141-1147. [Crossref]

20. Stolzenberg-Solomon RZ, Falk RT, Stanczyk F, Hoover RN, Appel LJ, et al. (2012) Sex hormone changes during weight loss and maintenance in overweight and obese postmenopausal African-American and non-African-American women. Breast Cancer Res 14: R141. [Crossref]

21. Ferrini RL, Barrett-Connor E (1998) Sex Hormones and age: a cross-sectional study of testosterone and estradiol and their bioavailable fractions in community-dwelling men: American Journal of Epidemiology 147: 750-754. [Crossref]

Copyright: (2019 Buynak RJ. This is an open-access article distributed under the terms of the Creative Commons Attribution License, which permits unrestricted use, distribution, and reproduction in any medium, provided the original author and source are credited. 